This is a non-peer reviewed EarthArXiv preprint version of the manuscript that has been submitted for publication in SCIENTIFIC DATA. Subsequent versions of this manuscript may have slightly different content. If accepted, the final version of this manuscript will be available via the peer reviewed publication DOI link.

Agricultural Sandbox NL: A national-scale database of parcel-level, processed Sentinel-1 SAR data Vineet Kumar ${ }^{1,2 *}$, Manuel Huber ${ }^{3}$, Bjorn Rommen ${ }^{3}$, Susan C. Steele-Dunne ${ }^{1}$

${ }^{1}$ Department of Geoscience and Remote Sensing, Delft University of Technology, Delft, 2600AA, The Netherlands

${ }^{2}$ Department of Water Management, Delft University of Technology, Delft, 2600AA, The Netherlands ${ }^{3}$ European Space Agency (ESA/ESTEC), Noordwijk, 2201AZ, The Netherlands

*Corresponding Author: Vineet Kumar (V.Kumar-1@tudelft.nl) 


\title{
Agricultural Sandbox NL: A national-scale database of parcel-level, processed Sentinel-1 SAR data
}

\author{
Vineet Kumar ${ }^{1,2, *}$, Manuel Huber ${ }^{3}$, Björn Rommen ${ }^{3}$, and Susan C. Steele-Dunne ${ }^{1}$ \\ ${ }^{1}$ Department of Geoscience and Remote Sensing, Delft University of Technology, Delft, 2600AA, The Netherlands \\ ${ }^{2}$ Department of Water Management, Delft University of Technology, Delft, 2600AA, The Netherlands \\ ${ }^{3}$ European Space Agency (ESA/ESTEC), Noordwijk, 2201AZ, The Netherlands \\ *corresponding author(s): Vineet Kumar (V.Kumar-1@tudelft.nl)
}

\& ABSTRACT

Synthetic Aperture Radar (SAR) data handling, processing, and interpretation are barriers preventing a rapid uptake of SAR data by application specialists and non-expert domain users in the field of agricultural monitoring. To improve the accessibility of Sentinel-1 data, we have generated a reduced-volume, multi-year Sentinel-1 SAR database. It includes mean and standard deviation of VV, VH and VH/VV backscatter, pixel counts, geometry, crop type, local incidence angle and azimuth angle 9 at parcel-level. The database uses around 3100 Sentinel-1 images (5 TB) to produce a 12 GB time series database for approximately 770,000 crop parcels over the Netherlands for a period of three years. The database can be queried by Sentinel-1 system parameters (e.g. relative orbit) or user application-specific parameters (e.g. crop type, spatial extent, time period) for parcel level assessment. The database can be used to accelerate the development of new tools, applications and methodologies for agricultural and water related applications, such as parcel-level crop bio-geophysical parameter estimation, inter-annual variability analysis, drought monitoring, grassland monitoring and agricultural management decision-support.

\section{Background \& Summary}

Synthetic Aperture Radar (SAR) satellite sensors provide Earth Observation (EO) data without being hindered by weather or solar illumination. The interaction of radar (microwave) signals with the surface depends on the properties of radar system as well as the target characteristics. In particular, changes in the geometric and dielectric properties of targets are associated with the backscattered signal received by a SAR system for a given resolution cell ${ }^{1}$.

The Copernicus Sentinel-1 SAR mission is a joint initiative of the European Space Agency (ESA) and the European Commission (EC) launched to image the global landmass, coastal zones, sea ice and polar areas at high geometric and radiometric resolutions. The mission comprising two identical satellites, Sentinel-1A (launched 3 April 2014) and Sentinel-1B (launched 25 April 2016) offers 6 day repeat cycle and acquires data at $5.405 \mathrm{GHz}$ (C-band) ${ }^{2}$. The operational interferometric wide (IW) mode provides dual-pol (VV+VH or $\mathrm{HH}+\mathrm{HV}$ ) data for a $250 \mathrm{~km}$ swath, with incidence angle variations from $30^{\circ}$ to $46^{\circ}$. By combining multiple orbital tracks from ascending and descending passes, it is possible to obtain Sentinel-1 data every 1-2 day in Europe ${ }^{3}$.

The large data volumes associated with SAR data create a need for high performance computing and data storage facilities for service providers as well as data users. For example, typical unzipped Sentinel-1 IW Single Look Complex (SLC), and Ground Range Detected (GRD) products ( $250 \mathrm{~km}$ by $175 \mathrm{~km}$ ) have data volumes of around $7 \mathrm{~GB}$ and $1.6 \mathrm{~GB}$ respectively. Recently, it has been reported that around 6 million Sentinel-1 data products, with a data volume of 10 petabytes were generated by the end of $2020^{4}$.

Due to these large data volumes, conventional methods of satellite data distribution, download, storing and processing may pose serious challenges to users who want to process imagery with local computing resources ${ }^{5}$. Regional and national-level monitoring applications such as agriculture, forestry, wetlands, urban area and infrastructure monitoring where past, requiring present and future dense time-series of SAR data necessitate the use of distributed computing platforms with integrated data repositories.

Cloud computing infrastructures such as Google Earth Engine (GEE) and Amazon Web Services (AWS) hosting the pre-processed historical and recently acquired satellite datasets ${ }^{6-8}$ have been enabled to ingest (amongst others) Copernicus Sentinel datasets allowing ease of data access and further processing. Google Earth Engine (GEE) is a cloud-based platform that archives a range of openly-available geospatial data and provides users access to high performance parallel computing with an internet-based application programming interface (API). GEE contains several satellite, thematic, vector, demographic and climate datasets ${ }^{9}$. The computing capabilities of GEE obviate the need for users to download, store and process high volume 
spatio-temporal data and ensure that this can be done in a fast and efficient manner ${ }^{10}$. The utility of the GEE platform has been shown in many studies involving large scale land cover dynamics mapping ${ }^{11,12}$, crop assessment and classification at various scales ${ }^{13-18}$, yield mapping ${ }^{19}$, grassland monitoring ${ }^{20}$ global urban land mapping ${ }^{21}$, drought assessment ${ }^{22}$, snow depth variability ${ }^{23}$ and many others. A detailed review of GEE based publications for various thematic applications using GEE stored geo-spatial dataset has been summarized here ${ }^{24-26}$.

Spaceborne Earth observation (EO) assets provide crucial information content for crop monitoring at regional, national and global scales. Information on parcel level crop dynamics is essential to support decision-making by farmers, agri-advisors, water boards and agro-farm industries. Advisors and grower organizations provide information to the farmers at parcel level. Parcel level spatio-temporal information from remote sensing observations facilitates the sustainable use of resources as well as precision farm management strategies ${ }^{20,27-29}$. Due to the burden of data storage and processing, SAR-based studies are typically limited to the smaller study areas. However, open availability of Sentinel-1 SAR ground range detected (GRD) products in GEE enable to rapid production of parcel-level temporal signatures.

The aim of the Agricultural SandboxNL is to improve the accessibility of Sentinel-1 SAR data for application experts interested in adapting or developing tools to use Sentinel-1 for agricultural monitoring. The Netherlands is ideally suited to the development and testing of such methodologies. Geospatial data assets and statistics are openly available via Publieke Dienstverlening Op de Kaart (PDOK) $)^{30}$ and Statistics Netherlands $(\mathrm{CBS})^{31}$. In addition, satellite from commercial providers are acquired regularly, and made freely available via the Netherlands Satellite Data Portal ${ }^{32}$. The objective of this study is to produce an interactive database that contains information extracted from multi-temporal Sentinel-1 SAR datasets at parcel level. The output database will support a range of SAR data uses for operational purposes with a demonstration of spatially tagged parcel level backscatter scalable at various administrative levels. This will reduce the burden of processing and extraction of large volume of Sentinel-1 SAR data for expert and non-expert data users.

\section{Methods}

The methods section is divided into four subsections: 1) Data sources describes the source and characteristics of the input vector and SAR remote sensing datasets; 2) Data processing details the functionalities implemented in GEE for SAR and vector data aggregation and processing; 3) Agricultural SandboxNL database generation describes how the GEE processed Sentinel-1 GRD images are converted into an interactive user-ready agricultural crop parcel-level database; 4) Sentinel-1 interferometric coherence describes how Single Look Complex (SLC) data are processed in SNAP and imported into GEE to generate a sample for the parcel-level database. The workflow adopted to generate the database is illustrated in Figure 2.

\section{Data sources \\ Parcel data and attributes}

A wide range of digital geospatial datasets in The Netherlands are openly accessed and hosted at the PDOK server under creative common licences (CC-BY-4.0). In Agricultural SandboxNL, annual Basisregistratie Gewaspercelen (BRP, Crop Parcel Base Register) and administrative layers such as province, municipality are used as base vector data ${ }^{33}$. The BRP dataset consists of the location of agricultural parcels, coupled with the cultivated crop name, unique parcel identifier and geometry. In total 312 distinct cultivated crops are defined in the BRP dataset. The boundaries of the agricultural parcels are based on Agrarisch Areaal Nederland (AAN, Agricultural Area of The Netherlands) ${ }^{33}$. The landholders of the parcels provide crop parcel boundaries every year, tagged with the main cultivated crop name. Since 2009, a draft data set with the reference date of 15 May is generated for each year and uploaded annually at PDOK web server. In this work, BRP vector data for the years 2017, 2018 and 2019 were used.

\section{Sentinel-1 SAR data}

GEE contains calibrated and ortho-rectified GRD products of Sentinel-1 SAR data, details of which are provided in Table 1. GEE-ingested Sentinel-1 data is pre-processed in the Sentinel Application Platform (SNAP) SAR data processing toolbox ${ }^{34}$ using the following steps: 1) Metadata update using restituted orbit files 2) Border noise removal 3) Thermal Noise Removal 4) Radiometric calibration 5) Terrain correction using SRTM30 or ASTER Digital Elevation Model (DEM) ${ }^{35}$. The final terrain-corrected Sigma Nought $\sigma^{0}$ backscatter values are available to fetch on a linear or logarithmic (dB) scale. All images also include an additional 'angle' band that contains the approximate viewing incidence angle. SRTM 30 meter v3 DEM product provided by NASA is used as an auxiliary data to generate the azimuth and local incidence angle band for Sentinel-1 SAR images. In GEE, Sentinel-1 GRD products are available over the globe since October 2014. The current version of the Agricultural SandboxNL database includes three years of Sentinel-1A/B images. Data from 2017 onwards are used due to the higher temporal resolution following the launch of Sentinel-1B in 2016. Sentinel-1 passes in 8 different relative orbits (RO) over the Netherlands in ascending (evening) and descending (morning) tracks. However, 6 RO (Ascending $(88,161,15)$ and Descending $(37,110,139)$ ) cover almost the entire geographical extent of the Netherlands (Figure 1), so these were used here . 


\section{Data processing Vector data processing}

The downloaded BRP vector layers from the PDOK server has an Amersfoort/RD New projected coordinate system (EPSG=28992), so those needed to be converted to geographical coordinates (EPSG: 4326) to be imported as a vector data asset in GEE platform. A spatial buffer of $-10 \mathrm{~m}$ is applied to the parcel polygons in the BRP data to remove edge effects due to e.g. parcel ridges, water channels, roads, building shades, adjacent fields. This is illustrated in Figure 3(a). Parcels smaller than $100 \mathrm{~m}^{2}$ are excluded from the database as the application of the buffer would make them prohibitively small. The centroid of each buffered parcel is calculated in GEE and added as an extra feature property to the BRP vector data to prevent ambiguity when parcels span administrative boundaries.

Figure 3(a) shows all 772,565 BRP parcels classified into 5 major land cover classes for the year 2019. It indicates that grassland is the dominant cover type, accounting for $66 \%$ of all parcels. Figure 3(b) shows the spatial distribution of the main summer crops in the Netherlands, namely silage maize, consumption potato, sugar beet and sow onions. Collectively, these crops account for 110,000 parcels.

\section{Sentinel-1 SAR data processing}

A set of processing steps are applied to the Sentinel-1 data in GEE before extracting the values for the database.The metadata of Sentinel-1 data at linear scale $S 1 \_G R D \_F L O A T$ are filtered for system parameters such as IW acquisition mode, overpass type (ascending/descending), 6 different $\mathrm{RO}$ and dual $(\mathrm{VV}+\mathrm{VH})$ polarization in space by using administrative boundaries and in time for each 2017, 2018 and 2019 year separately. Next, the following functions are implemented in GEE on the filtered Sentinel-1 image collections.

\section{Border Noise Removal}

The transformation of Sentinel-1 level-0 to level-1 products using the ESA Instrument Processing Facility (IPF) (16 $^{36}$ leads to generation of artifacts such as "no-value" and "very low values" pixels due to azimuth/range compression and the sampling start time window offsets ${ }^{5}$ at the range and azimuth borders of the image data. The effect of border noise pixels has been resolved in IPF v 2.90 and later versions (after March 2018 products in GEE ${ }^{35}$ ). However, for earlier IPF versions, these effects can be observed at the borders of GRD data products (Figure 4). In the Agricultural SandboxNL, imagery from 2017,2018 and 2019 are considered for database generation. Hence, removal of these "no-value" and "very low values" border pixels is an important step to ensure that the data record is consistent for multi-year studies. Outside the GEE platform, the SNAP toolbox provides an operator Sentinel-1 Remove GRD border noise that uses de-noising vectors on the VV or HH-pol data by first setting dark pixels to zero values and then removing them using a thresholding approach ${ }^{36}$. Several approaches, namely anomaly detection based on statistical interquartile range ${ }^{5}$, bidirectional sampling based ${ }^{5}$ and mathematical morphology ${ }^{37}$ based methods are introduced in the literature. However, their implementation in GEE is more challenging due to their complexity and computational efficiency for large volume of data. Within the GEE environment, previous studies have applied single value backscatter thresholds ${ }^{38}$, and median composite filter ${ }^{39}$ on the entire image. However, these might affect other pixel values in addition to the border pixels.

To overcome the border noise issue in the Agricultural SandboxNL dataset, we have employed a simple threshold technique on the outer edges (near and far range) of the image. This means the threshold was only applied on pixels with an approximate viewing incidence angle of $<30$ degrees and $>45$ degrees. To remove pixels with very low values a threshold value of 0.003 linear or $-35 \mathrm{~dB}$ was applied. Other advantages of this simple technique are the simple implementation and the computational efficiency as it is only applied to the image border regions. This mask is created using VV polarization values only, and applied over the image collection for both VV and VH pol data. This avoids any incorrect removal of low pixel values in cross-polarization. To have consistent results, the masking was executed for all images, including 2019. As, the approach is based on simple value masking, there are chances that some $N a N$ or very low data values are present at border pixels. Those $N a N$ pixels are removed in the later steps of database creation. An example illustrating the effect of border noise removal on January 2018 (RO-37) is shown in Figure 4.

\section{Local incidence angle calculation}

Radar backscatter depends on both system and target characteristics. Due to the influence of surface geometry on radar backscatter, local incidence angle (LIA) needs to be taken into account for the retrieval of geophysical variables of interest ${ }^{40}$. Calculation of local incidence angle requires geometric information from the viewing radar and the terrain ${ }^{40}$. Terrain geometry parameters, namely slope and aspect values, are extracted from the SRTM30 DEM which is available in GEE. Radar look direction is defined by the incidence angle and range-look direction. In this study, approximate viewing incidence angle information is directly used as captured by Sentinel-1 GRD data in GEE ${ }^{41}$. However, range direction information is not directly available with Sentinel-1 data products, hence it is calculated from Sentinel-1 image geometry by calculating azimuth information as discussed in ${ }^{42,43}$. Both LIA and azimuth angle are calculated separately for ascending and descending track images. 


\section{Sentinel-1 backscatter observable}

Parcel-averaged backscatter coefficients in VV and VH are included in the Agricultural SandboxNL database. In addition, the parcel-averaged cross-polarization ratio $(\mathrm{VH} / \mathrm{VV})$ is calculated. The use of this ratio is becoming increasingly common in agricultural monitoring as it reduces the influence of soil moisture on the retrieval of vegetation parameters ${ }^{44-47}$. All the images are stacked and the $\mathrm{VH}, \mathrm{VV}$ and $\mathrm{VH} / \mathrm{VV}$ backscatter pixels within the parcels are averaged and the standard deviation within each parcel is obtained. This results in a table containing the mean and standard deviation of VV, VH and VH/VV, LIA, azimuth angle and pixel counts at parcel level with unique OBJECTID. Note that a speckle noise removal filter is not implemented during the backscatter information extraction because the backscatter values are spatially averaged over the parcel.

\section{Agricultural SandboxNL database generation}

The border noise removal, local incidence angle determination and cross-ratio calculations are implemented in GEE environment. The standard programming language of GEE is java script. In this study the GEE Python API and additional python packages such as geemap ${ }^{48}$ are used to utilize the computational capability of GEE and its tools in a python environment. The geemap package, in particular, makes it possible to directly translate any given java script into python syntax.

The database generation involves several steps. First, all BRP and administrative boundary shapefiles need to be uploaded manually as an asset in GEE and the elevation data (SRTM DEM) is imported directly from GEE. The second step is to control and manage the automation of the extraction process. This step poses several limitations and challenges. First, GEE has a memory threshold for each user to reduce overload of the system, which means only a selection of parcels can be processed and downloaded at a time. Another constraint is a discrepancy in GEE output values depending on the number and spatial extent of selected parcels. To circumvent these issues, the data were downloaded per municipality of The Netherlands to keep the feature collection extent relatively constant and localized. In addition, a maximum of 500 parcels were processed at a time to maintain a systematic mining process, and avoid memory limitations in the GEE. The border noise removal, cross-ratio determination and LIA calculation are applied for every batch of 500 parcels and the results are stored as a csv file on the local machine. The processing, flagging and cleaning of the data is explained in the Data Records section.

\section{Sentinel-1 Interferometric coherence}

The primary dataset of the Agricultural SandboxNL is the Sentinel-1 radar backscatter because the value of backscatter has been widely demonstrated in mapping and monitoring activities. Several recent studies have highlighted the potential value of interferometric coherence for crop type mapping ${ }^{49}$, crop emergence, harvest detection ${ }^{45,50}$ and grassland monitoring ${ }^{51}$. However, GEE does not host the Sentinel-1 SLC products required to produce interferometric coherence ${ }^{35}$. In order to provide a sample of interferometric coherence data, the SLC images (RO 110) for the province of Flevoland were downloaded from the Copernicus Open Access Hub ${ }^{52}$ and processed offline in the SNAP toolbox for the 2019 growing season.

The coherence between two SLC images $S 1$ and $S 2$ is defined in equation 1. A standard processing chain ${ }^{53}$ is applied in the SNAP toolbox to calculate the 6-day InSAR coherence. The spatially-averaged parcel level interferometric coherence values are calculated from the 6-day pairs of Sentinel-1 in VV and VH polarizations. A total of 32 pairs of Sentinel-1 SLC products are analyzed during the period of April to October 2019. Data are processed for the province of Flevoland as the crop types in this area include structurally different crops such as potato, sugar beet, maize, and winter wheat.The SNAP generated coherence images in .tiff format are then ingested into GEE to create parcel-level data, in a format consistent with the backscatter data.

$$
\gamma=\frac{\left|\left\langle\mathbf{S}_{1} \mathbf{S}_{2}^{*}\right\rangle\right|}{\sqrt{\left\langle\mathbf{S}_{1} \mathbf{S}_{1}^{*}\right\rangle\left\langle\mathbf{S}_{2} \mathbf{S}_{2}^{*}\right\rangle}}
$$

\section{Data Records}

The annual database consists of spatially averaged VV, VH and VH/VV backscatter values, corresponding standard deviation, viewing incidence angle, local incidence angle, azimuth angle and pixel count for each parcel. Every crop parcel has a unique field ID (OBJECTID), which is used as an index to sort and process the data. The data cleaning involves identification of parcels with no data due value due to their size smaller than $100 \mathrm{~m}^{2}$.

After cleaning the data, each parcel is stored separately in a data frame containing the time series of all the above-mentioned extracted parameters. A dictionary of all of these data frames is temporarily stored together with static information in form of a pickle file. Static information includes attributes of the BRP vector data such as parcel geometry, area, centroid, crop type and flag information. Finally, the pickle dataset is transformed to a netCDF file to reduce the data size and to provide a universal data format for the scientific user community. Static information about the parcel geometry and crop type is not saved in the $n e t C D F$ file but can be accessed via the BRP files. Table 2 gives an overview of variables stored in the final netCDF product. The datasets are provided per province and per year. A technical validation was conducted to flag all parcels which lie on 
the azimuth and range boundary of the image. Details about this validation and flagging process are discussed in the section "Technical Validation".

The Agricultural SandboxNL database supplemented by BRP parcel vector data is published for the public use, can be found on the 4TU Centre for Research Data repository (DOI:https://doi.org/10.4121/14438750). The readme files can be consulted for explanations of the data records, query and visualizations. Table 3 indicates the total no. of BRP parcels, Sentinel-1 GRD images processed and size of the output database annually.

\section{Technical Validation}

The section describes the validation of the Agricultural SandboxNL database against the SNAP extracted Sentinel-1 backscatter values, and the flagging of the BRP parcels which are at the azimuth and range boundary of image geometry. The purpose of the validation is to control that no alteration of the data occurred during the data processing steps in GEE and python. The blue highlighted boxes in Figure 2 indicate all processing steps to be validated. SNAP V8.0 toolbox ${ }^{34}$ is used for this validation as it was also utilized by GEE to create the Sentinel-1 SAR GRD product. To guarantee a sound comparison the same pre-processing steps as in $\mathrm{GEE}^{41}$ were applied onto the Copernicus Open Access Hub ${ }^{52}$ Sentinel-1 SAR GRD data. The SNAP-processed backscatter output values are therefore considered as a reference. The validation is performed over the province of Flevoland, which consists of approximately 16,500 crop parcels. Sentinel-1 descending images in RO-37 were downloaded on 15-04-2019, 20-07-2019 and 18-10-2019 to span a typical summer crop season. Parcel conditions are generally heterogeneous in April, due to the bare soil surface and ongoing field preparation activities. July corresponds to the period with maximum crop cover and biomass. By October, most crops have been harvested. The GRD images processed in SNAP were imported to GEE in .tiff file format to apply the field buffer zones and to extract parcel-averaged VH, VV backscatter values. Finally, the validation was performed between the SandboxNL database and the SNAP toolbox processed images, by comparing the parcel-averaged backscatter values. The extracted backscatter values are highly correlated with those directly from SNAP, (Figure 5) with a goodness of fit $\left(\mathrm{R}^{2}\right)$ of at least $\sim 0.99$ for all dates in both the polarizations. Despite the high $\mathrm{R}^{2}$ values, some deviations are visible between GEE and SNAP extracted backscatter. These deviations have already been reported in ESA SNAP toolbox community forum ${ }^{54}$. The reported difference in the $\mathrm{R}^{2}$ values between the three dates may be due to the complexity and noise characteristics of the backscattered signal, influenced by differences in soil moisture, crop growth stage, rainfall and other environmental factors and potential non-linear dependence of the backscatter values (with their location in the image) due to additional processing of the data within GEE (e.g. tiling approach).

\section{Data quality and flags}

Figure 1 shows that Sentinel-1 tracks may not be exactly covering the entire Netherlands administrative boundaries. In range direction, RO 38 and 88 cover a major part of the geographic extent of the Netherlands whereas RO 110, 139, 161 and 15 cover approximately half of the total geographic area. It is important to flag the parcels lying at the near and far range of the image borders in range direction as the pixel count in these parcel will vary depending on the relative orbit. The average backscatter and standard deviation are those calculated when the parcel is completely within a Sentinel-1 image. On dates when the parcel is only partially covered, a data quality flag is assigned to parcels in the database as shown in Figure 6.

The along-track dimensions of a data take obviously depend on the Sentinel-1 observation scenario that dictates that over Europe all passes are consistently acquired. The full along-track product from a single acquisition is segmented into slices with unique time stamps for data distribution ${ }^{55}$. In the SNAP toolbox, the $S$ - 1 Slice Assembly function seamlessly combines these sliced products into an assembled product without any data gap or discontinuity. However, this function is currently not available in GEE. In the Agricultural SandboxNL database generation, some crop parcels may be covered by two slices. To handle this continuity, the parcel average is obtained using a weighted average based on the pixel counts in each slice. The time-stamp of the slice containing most pixels is used as the data-stamp in the database. These azimuth border parcels are flagged as shown in Figure 6.

In the processing chain every orbit is treated separately and the data from all orbits are concatenated at the end. The flagging methods compare the absolute difference between the unique list of dates and the actual list of dates. If the unique list of dates is smaller than the actual list of dates, the parcel gets flagged with 1, indicating that it is on the azimuth border of the image. Near and far range border effects have daily unique observations and but have different pixel counts. These filter conditions are applied on the fields at the outer edges of the image $(<30.1$ and $>44.9$ degrees of the approximate viewing incidence angle), and the parcels are flagged with a value of 2 . The flag information is stored per orbit in the general parcel information.

Sentinel-1 acquires data in IW mode using the Terrain Observations with Progressive Scan (TOPS) multi-swath observation technique. After application of the thermal noise filter, some small radiometric jump could still be observed in some Sentinel-1 GRD images, especially at the subswath edges. This sometimes results in a visible discontinuity at the subswath edge in the generated database, particularly in VH-pol and cross-pol ratio images. One explanation for this undesired effect could be a small unresolved residual calibration bias in elevation that is further amplified when using VH/VV ratios. Figure 7 shows that 
the cross-pol ratio is influenced by this effect at interswath positions in all the shown images, i.e. generated database, GEE Image and SNAP extracted image of 17 July 2019 data in RO-88. Although in this particular case (mid July, well-developed crops), the backscatter at both co- and cross-polarization should not be influenced by thermal noise, slight inconsistencies in the applied thermal noise removal procedure (equivalent in both GEE and SNAP) could also have led to such effect. Therefore, such residual calibration or processing bias is in the Sentinel-1 data products themselves, and not an artifact of the database generation. Nonetheless, the user should be mindful of this artifact when interpreting backscatter values in these areas.

\section{Usage Notes}

The Agricultural SandboxNL database is freely available at the 4TU Centre for Research Data repository (4TU DOI: https : //doi.org/10.4121/14438750) under a CC BY-SA 4.0 licence. The database is stored annually in netCDF format for the 12 provinces of the Netherlands. In addition, the re-projected and spatially buffered BRP parcel boundaries for the Netherlands for all the three years 2017, 2018 and 2019 are provided as shapefiles. In general, two types of information are stored: 1) Static Information and 2) Dynamic Information. Static information refers to the unique OBJECTID, parcel centroid, polygon, crop type and data flags. Dynamic information consist of all data variables that change in time, i.e. VV, VH, VH/VV and local incidence angle. Depending on the needs of the users, data can be selected and filtered using either static or dynamic constraints. In the example python code, provided along with this publications repository, different scenarios are presented in which the data are visualized per longitude and latitude, crop type, OBJECTID, relative orbit and specific time stamp. The unique OBJECTIDs per parcel allow to join data across datasets. This gives users great flexibility to manipulate the data spatially and temporally to meet the their specific requirements. In this section, we demonstrate the usage of the Agricultural SandboxNL database to query and visualize data in space and time at national and province level using VH/VV cross-pol ratio. Additionally, Sentinel-1 SLC data derived coherence maps are shown over the Flevopolder region to showcase the InSAR coherence data included in the dataset.

In Figure 8, an example is provided in which the database has been queried in time. It shows the parcel level cross ratio VH/VV (CR) over the entire Netherlands for three dates in 2019. Querying the data to extract an image of all of the Netherlands on dates before, during, and after the growing season allows the user to see that CR is clearly related to biomass ${ }^{44,47}$. CR values are low on 12 April 2019 compared to other dates as this the general crop planting time in the Netherlands. Provinces dominated by arable crop parcels such as Flevoland, Noord-Brabant, Drenthe, Limburg and Zeeland show CR values in the range of -12 to $-8 \mathrm{~dB}$ as the soil is mostly bare and being prepared for planting. Most parcels in Friesland, Gelderland, Overijssel, Utrecht and Zuid-Holland are grassland (Perennial ryegrass). The CR values for the grasslands are in the range of -8 to $-5 \mathrm{~dB}$ on 12 April 2019 data. Figure 8 shows that CR values on 20 July 2019 are higher than those on 12 April 2019 due to the peak vegetation biomass stage of the summer crops. For the summer crops such as maize, potato, onion, and barley, these values range from -8 to $-5 \mathrm{~dB}$ due to strong scattering from the vegetation. Figure 8(b) on 18 October 2019, typically indicates the crop harvest period for summer crops in the Netherlands.

In addition to querying the data by date to produce spatial maps, dynamic radar backscatter ( $\mathrm{VV}, \mathrm{VH}$ and $\mathrm{VH} / \mathrm{VV}$ ) information can be extracted to produce time series per crop type and per orbit. Data can be analyzed individually at parcel-level or aggregated using administrative boundaries. We have demonstrated one such case of querying the database used by extracting the cross-ratio values for four major summer crops i.e. maize, onion, potato, sugar beet. The CR values are extracted for the year 2019 in descending $37 \mathrm{RO}$ and aggregated by province in Figure 9. From the start of the year until the emergence, the Sentinel-1 backscatter observable (VV, VH and VH/VV) are mainly dependent on the attributes of exposed soil surface such as texture, roughness, moisture content and orientation.

The CR ratio decreases after March, probably due to changes in roughness. Standard deviation is high during the bare soil period due to differences in row orientation. The standard deviation in CR values for potato is much higher than the other crops due to the deep ridges in the potato fields. The $\mathrm{CR}$ values increase after emergence of due to the increase of in biomass. The VH/VV ratio increases because VH backscatter is more sensitive to the vegetation than the VV backscatter. CR values start plateauing as the crop reaches maximum height for vertically oriented stalk crops and canopy coverage is maximum for broadleaf crops. The CR values start sharply decreasing from their peak at the ripening of corn and onion due to the reduction in vegetation water content and the start of harvesting. Unlike maize and onion, CR values for sugar beets decrease gradually as harvest may occur between mid-September to late November.

Finally, Figure 10 shows three samples of the interferometric coherence data included in the dataset. These images highlight the high coherence $(>0.4)$ at the early transplanting stage and harvesting period when most of the backscatter response is from the exposed soil surface in VV polarization, and lower values $(<0.3)$ during the vegetative phase of crop growth. These are due to crop growth, water content changes and motion of the canopy in the wind. This allows the user to explore the potential value of transitions in interferometric coherence as indicators of crop emergence and harvest dates.

The ease with which users can download, manage and query the data in space, time and per system parameter means that this database is highly accessible for new users of SAR data. In addition, reducing the data volume from $1.6 \mathrm{~TB}$ per year to 4 
GB, and providing processed data means that expertise in SAR processing software, and access to high-performance computing facilities is no longer a limiting factor for research and applications development. It is hoped that creating this capacity to explore and experiment with three years of SAR data for 770,000 agricultural parcels, on an almost daily basis, will stimulate the development of new techniques and applications of SAR data in agricultural applications.

\section{Code availability}

Python code to access, query, visualize and analyze the Agricultural SandboxNL database is distributed, with the dataset and accompanying documentation.

\section{References}

1. Ulaby, F. Radar response to vegetation. IEEE Transactions on Antennas Propag. 23, 36-45, https://doi.org/10.1109/TAP. 1975.1140999 (1975).

2. Torres, R. et al. Gmes Sentinel-1 mission. Remote. Sens. Environ. 120, 9-24, https://doi.org/10.1016/j.rse.2011.05.028 (2012). The Sentinel Missions - New Opportunities for Science.

3. Sentinel-1 SAR user guide. https://sentinel.esa.int/web/sentinel/user-guides/sentinel-1-sar. Accessed: 2020-11-20.

4. Sentinel-1 Infographic. https://sentinel.esa.int/documents/247904/4603794/Sentinel-1-infographic.pdf. Accessed: 202103-01.

5. Ali, I. et al. Sentinel-1 data cube exploitation: Tools, products, services and quality control. Proc. Big Data Space 40-43 (2017).

6. Gorelick, N. et al. Google Earth Engine: Planetary-scale geospatial analysis for everyone. Remote. Sens. Environ. 202, 18 27, https://doi.org/10.1016/j.rse.2017.06.031 (2017). Big Remotely Sensed Data: tools, applications and experiences.

7. Jackson, K. R. et al. Performance analysis of high performance computing applications on the amazon web services cloud. In 2010 IEEE Second International Conference on Cloud Computing Technology and Science, 159-168 (2010).

8. Shelestov, A. et al. Cloud approach to automated crop classification using Sentinel-1 imagery. IEEE Transactions on Big Data 6, 572-582, https://doi.org/10.1109/TBDATA.2019.2940237 (2020).

9. Google Earth Engine Datasets. https://earthengine.google.com/datasets/. Accessed: 2019-07-01.

10. Mutanga, O. \& Kumar, L. Google earth Engine Applications. Remote. Sens. 11, https://doi.org/10.3390/rs11050591 (2019).

11. Huang, H. et al. Mapping major land cover dynamics in Beijing using all Landsat images in Google Earth Engine. Remote. Sens. Environ. 202, 166-176, https://doi.org/10.1016/j.rse.2017.02.021 (2017).

12. Midekisa, A. et al. Mapping land cover change over continental Africa using Landsat and Google Earth Engine cloud computing. PloS one 12, e0184926, https://doi.org/10.1371/journal.pone.0184926 (2017).

13. Liu, X. et al. Large-Scale Crop Mapping From Multisource Remote Rensing Images in Google Earth Engine. IEEE J. Sel. Top. Appl. Earth Obs. Remote. Sens. 13, 414-427, https://doi.org/10.1109/JSTARS.2019.2963539 (2020).

14. Phalke, A. R. et al. Mapping croplands of Europe, Middle East, Russia, and Central Asia using Landsat, Random Forest, and Google Earth Engine. ISPRS J. Photogramm. Remote. Sens. 167, 104-122, https://doi.org/10.1016/j.isprsjprs.2020.06.022 (2020).

15. Shelestov, A., Lavreniuk, M., Kussul, N., Novikov, A. \& Skakun, S. Exploring Google Earth Engine platform for big data processing: Classification of multi-temporal satellite imagery for crop mapping. frontiers Earth Sci. 5, 17, https://doi.org/10.3389/feart.2017.00017 (2017).

16. Xiong, J. et al. Automated cropland mapping of continental Africa using Google Earth Engine cloud computing. ISPRS J. Photogramm. Remote. Sens. 126, 225-244, https://doi.org/10.1016/j.isprsjprs.2017.01.019 (2017).

17. Mandal, D. et al. Sen4rice: A Processing Chain for Differentiating Early and Late Transplanted Rice Using Time-Series Sentinel-1 SAR Data with Google Earth Engine. IEEE Geosci. Remote. Sens. Lett. 15, 1947-1951, https://doi.org/10.1109/ LGRS.2018.2865816 (2018).

18. Singha, M., Dong, J., Zhang, G. \& Xiao, X. High resolution paddy rice maps in cloud-prone Bangladesh and Northeast India using Sentinel-1 data. Sci. data 6, 1-10, https://doi.org/10.1038/s41597-019-0036-3 (2019).

19. Jin, Z. et al. Smallholder maize area and yield mapping at national scales with Google Earth Engine. Remote. Sens. Environ. 228, 115-128, https://doi.org/10.1016/j.rse.2019.04.016 (2019). 
20. D'Andrimont, R., Lemoine, G. \& Van der Velde, M. Targeted grassland monitoring at parcel level using Sentinels, street-level images and field observations. Remote. Sens. 10, 1300, https://doi.org/10.3390/rs10081300 (2018).

21. Liu, X. et al. High-resolution multi-temporal mapping of global urban land using Landsat images based on the Google Earth Engine Platform. Remote. sensing environment 209, 227-239, https://doi.org/10.1016/j.rse.2018.02.055 (2018).

22. Sazib, N., Mladenova, I. \& Bolten, J. Leveraging the Google Earth Engine for drought assessment using global soil moisture data. Remote. sensing 10, 1265, https://doi.org/10.3390/rs10081265 (2018).

23. Lievens, H. et al. Snow depth variability in the Northern Hemisphere mountains observed from space. Nat. communications 10, 1-12, https://doi.org/10.1038/s41467-019-12566-y (2019).

24. Amani, M. et al. Google Earth Engine cloud computing platform for remote sensing big data applications: A comprehensive review. IEEE J. Sel. Top. Appl. Earth Obs. Remote. Sens. 13, 5326-5350, https://doi.org/10.1109/JSTARS.2020.3021052 (2020).

25. Tamiminia, H. et al. Google Earth Engine for geo-big data applications: A meta-analysis and systematic review. ISPRS J. Photogramm. Remote. Sens. 164, 152-170, https://doi.org/10.1016/j.isprsjprs.2020.04.001 (2020).

26. Wang, L. et al. A summary of the special issue on remote sensing of land change science with Google Earth Engine, https://doi.org/10.1016/j.rse.2020.112002 (2020).

27. Defourny, P. et al. Near real-time agriculture monitoring at national scale at parcel resolution: Performance assessment of the Sen2-Agri automated system in various cropping systems around the world. Remote. sensing environment 221, 551-568, https://doi.org/10.1016/j.rse.2018.11.007 (2019).

28. Kussul, N. et al. Parcel-based crop classification in Ukraine using Landsat-8 data and Sentinel-1A data. IEEE J. Sel. Top. Appl. Earth Obs. Remote. Sens. 9, 2500-2508, https://doi.org/10.1109/JSTARS.2016.2560141 (2016).

29. Sitokonstantinou, V. et al. Scalable parcel-based crop identification scheme using Sentinel-2 data time-series for the monitoring of the common agricultural policy. Remote. Sens. 10, https://doi.org/10.3390/rs10060911 (2018).

30. Publieke Dienstverlening Op de Kaart, PDOK. https://www.pdok.nl/. Accessed: 2021-04-23.

31. Statistics Netherlands, CBS. https://www.cbs.nl/en-gb. Accessed: 2021-04-23.

32. Netherlands Satellite Data Portal. https://www.satellietdataportaal.nl/. Accessed: 2021-04-23.

33. Dataset: Basisregistratie Gewaspercelen (BRP). https://www.pdok.nl/introductie/-/article/ basisregistratie-gewaspercelen-brp-. Accessed: 2020-10-25.

34. SNAP Toolbox. https://step.esa.int/main/download/snap-download/. Accessed: 2021-03-15.

35. Google Earth Engine guides: Sentinel-1 algorithms. https://developers.google.com/earth-engine/guides/sentinel1. Accessed: 01.12.2020.

36. Hajduch, G. \& Miranda, N. Masking "no-value" pixels on GRD products generated by the Sentinel-1 ESA IPF. Document Ref. MPC-0243 (2018).

37. Stasolla, M. \& Neyt, X. An operational tool for the automatic detection and removal of border noise in Sentinel-1 GRD products. Sensors 18, https://doi.org/10.3390/s18103454 (2018).

38. DeVries, B. et al. Rapid and robust monitoring of flood events using Sentinel-1 and Landsat data on the Google Earth Engine. Remote. Sens. Environ. 240, 111664, https://doi.org/10.1016/j.rse.2020.111664 (2020).

39. Rudiyanto et al. Automated near-real-time mapping and monitoring of rice extent, cropping patterns, and growth stages in Southeast Asia using Sentinel-1 time series on a Google Earth Engine platform. Remote. Sens. 11, https: //doi.org/10.3390/rs11141666 (2019).

40. O'Grady, D., Leblanc, M. \& Gillieson, D. Relationship of local incidence angle with satellite radar backscatter for different surface conditions. Int. J. Appl. Earth Obs. Geoinformation 24, 42-53, https://doi.org/10.1016/j.jag.2013.02.005 (2013).

41. Google Earth Engine Data Catalog, Sentinel-1 GRD. https://developers.google.com/earth-engine/datasets/catalog/ COPERNICUS_S1_GRD. Accessed: 23.04.2021.

42. Google Earth Engine Developer Group. Discussion on derivation of Local Incidence Angle from Sentinel-1 SAR data. https: //groups.google.com/forum/\#tprotectlkern-.1667em/google-earth-engine-developers/3-q0TEwa-Tk/h3J4havuBAAJ. Accessed: 2020-06-10.

43. Vollrath, A., Mullissa, A. \& Reiche, J. Angular-based radiometric slope correction for Sentinel-1 on Google Earth Engine. Remote. Sens. 12, https://doi.org/10.3390/rs12111867 (2020). 
44. Vreugdenhil, M. et al. Sensitivity of Sentinel-1 backscatter to vegetation dynamics: An austrian case study. Remote. Sens. 10, https://doi.org/10.3390/rs10091396 (2018).

45. Khabbazan, S. et al. Crop monitoring using Sentinel-1 data: A Case Study from The Netherlands. Remote. Sens. 11, https://doi.org/10.3390/rs11161887 (2019).

46. Veloso, A. et al. Understanding the temporal behavior of crops using sentinel-1 and sentinel-2-like data for agricultural applications. Remote. Sens. Environ. 199, 415-426, https://doi.org/10.1016/j.rse.2017.07.015 (2017).

47. Vreugdenhil, M. et al. Sentinel-1 cross ratio and vegetation optical depth: A comparison over Europe. Remote. Sens. 12, https://doi.org/10.3390/rs12203404 (2020).

48. Wu, Q. geemap: A python package for interactive mapping with Google Earth Engine. J. Open Source Softw. 5, 2305, $10.21105 /$ joss. 02305 (2020).

49. Mestre-Quereda, A., Lopez-Sanchez, J. M., Vicente-Guijalba, F., Jacob, A. W. \& Engdahl, M. E. Time-series of Sentinel-1 interferometric coherence and backscatter for crop-type mapping. IEEE J. Sel. Top. Appl. Earth Obs. Remote. Sens. 13, 4070-4084, https://doi.org/10.1109/JSTARS.2020.3008096 (2020).

50. Kavats, O., Khramov, D., Sergieieva, K. \& Vasyliev, V. Monitoring harvesting by time series of Sentinel-1 SAR data. Remote. Sens. 11, https://doi.org/10.3390/rs11212496 (2019).

51. Tamm, T., Zalite, K., Voormansik, K. \& Talgre, L. Relating Sentinel-1 interferometric coherence to mowing events on grasslands. Remote. Sens. 8, 802, https://doi.org/10.3390/rs8100802 (2016).

52. Copernicus Open Access Hub. https://scihub.copernicus.eu/dhus/\#/home. Accessed: 2019-11-10.

53. Braun, A. \& Veci, L. Sentinel-1 Toolbox: TOPS Interferometry Tutorial. http://step.esa.int/docs/tutorials/S1TBX\% 20TOPSAR\%20Interferometry\%20with\%20Sentinel-1\%20Tutorial_v2.pdf. Accessed: 2020-02-15.

54. Comparision of GRD Products. https://forum.step.esa.int/t/comparison-of-grd-products-original-grd-slcl -converted-to-grd-grd-from-google-earth-engine/12391. Accessed: 2021-03-20.

55. Sentinel-1 SAR Product Slices. https://sentinels.copernicus.eu/web/sentinel/user-guides/sentinel-1-sar/data-formats/ product-slices. Accessed: 2021-03-20.

\section{Acknowledgements}

The authors would like to thank European Space Agency (ESA) for supporting the Agricultural SandboxNL project (Contract No. 5001025295).

\section{Author contributions statement}

V.K., B.R. and S.S.D conceptualized the project. V.K. and M.H. wrote the code and generated the database. V.K. and M.H. performed the validation. B.R. and S.S.D. provided the supervision and guidance. All authors analyzed the database, contributed in writing the manuscript.

\section{Competing interests}

The authors declare no competing interests.

\section{Figures \& Tables}




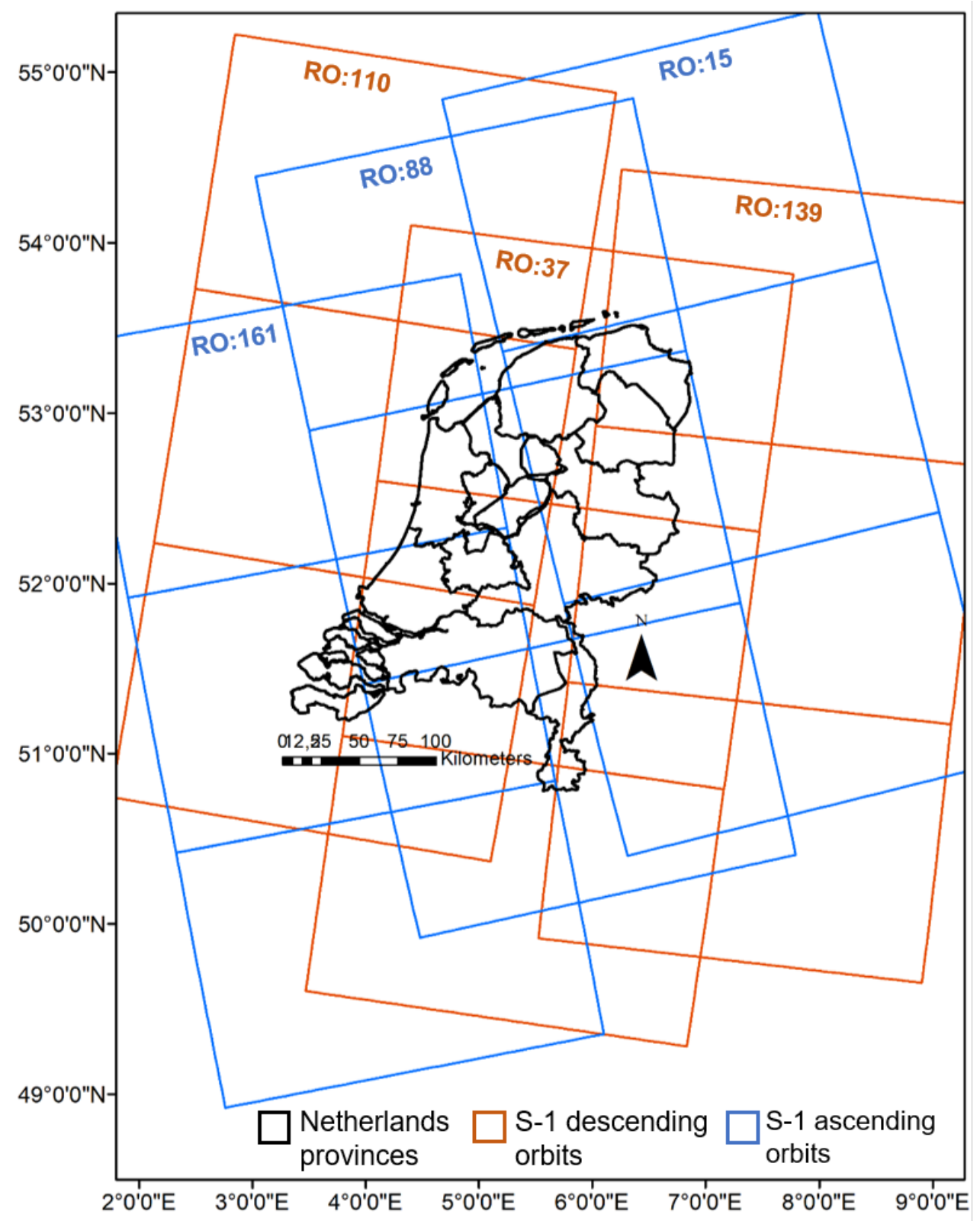

Figure 1. Sentinel-1 coverage over the Netherlands based on six relative orbits (RO). The blue polygons indicate ascending orbits $(161,88,15)$ and the orange indicate descending orbits $(139,110,37)$. 


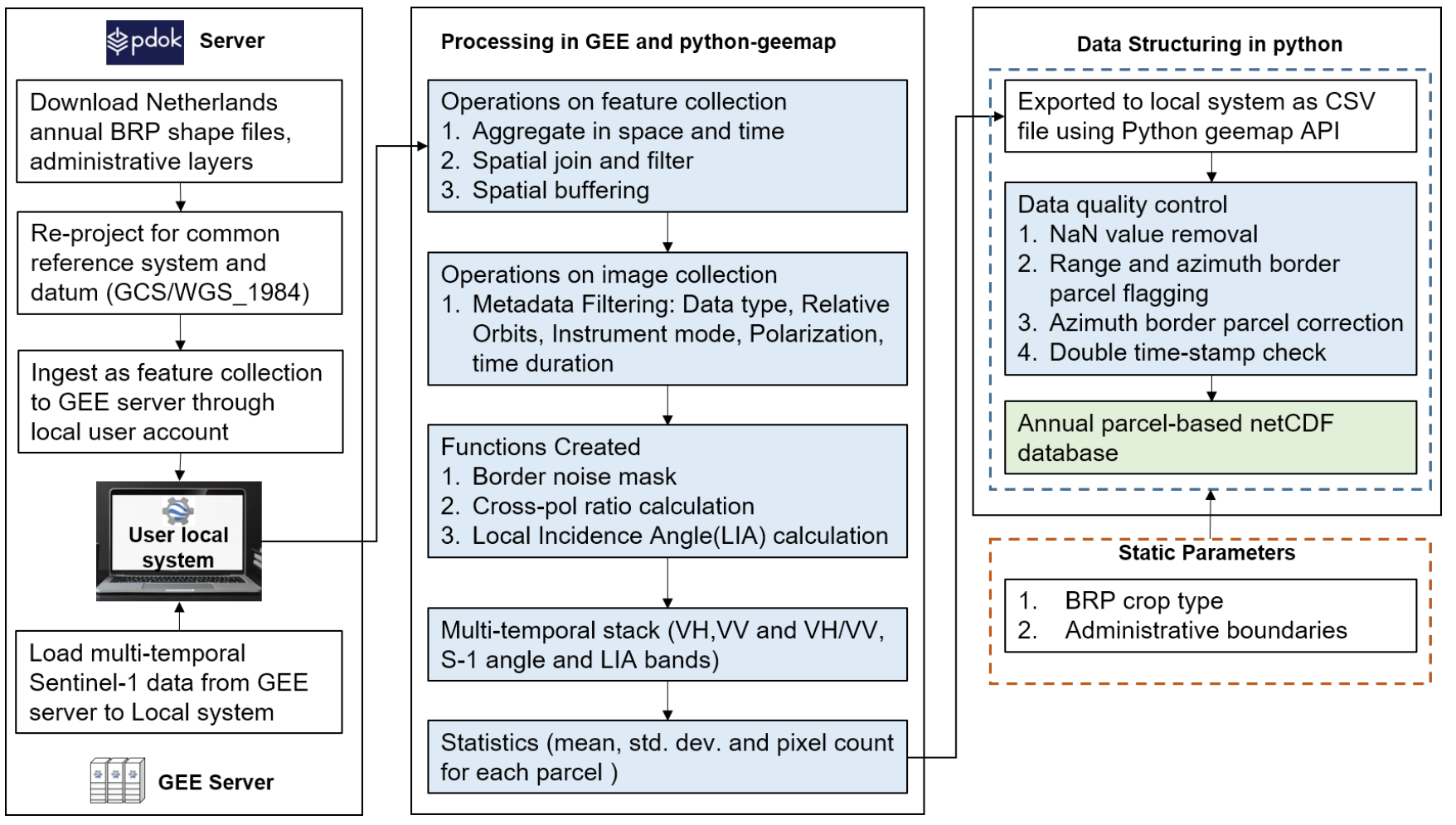

Figure 2. Proposed processing steps in the Agricultural SandboxNL database creation. The blue highlighted boxes indicate all processing steps applied onto the GRD data in order to create the database. The green box indicates the final dataset. 


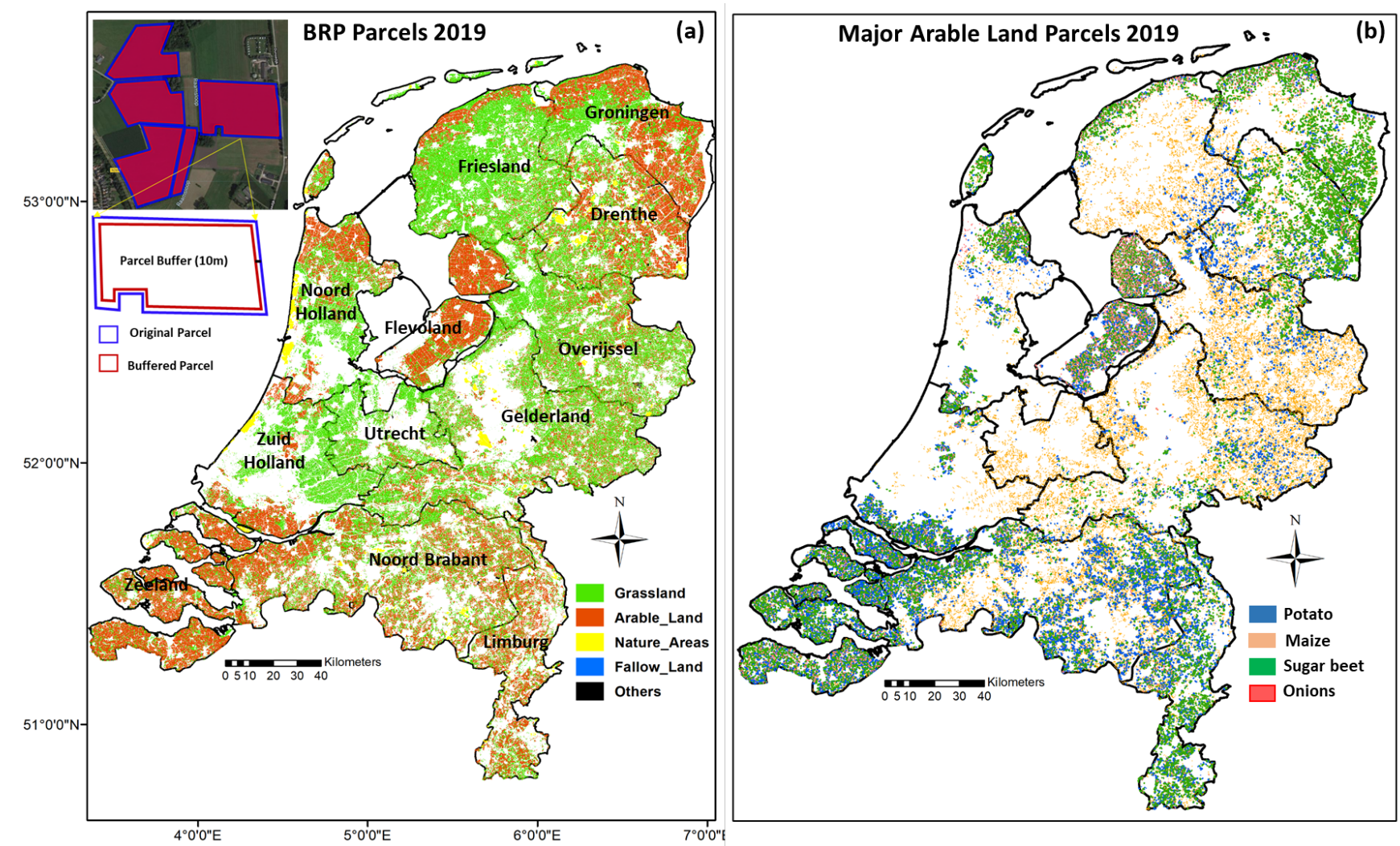

Figure 3. Two example images illustrating the BRP vector data for major crops of the Netherlands. The left image indicates crop classes such as grassland, arable land, nature areas and fallow land, whereas the right image shows the major arable crop types (potato, maize, sugar beet and onions). The inset image in the upper-left corner of the left figure shows the $10 \mathrm{~m}$ spatial buffer applied on each parcel.

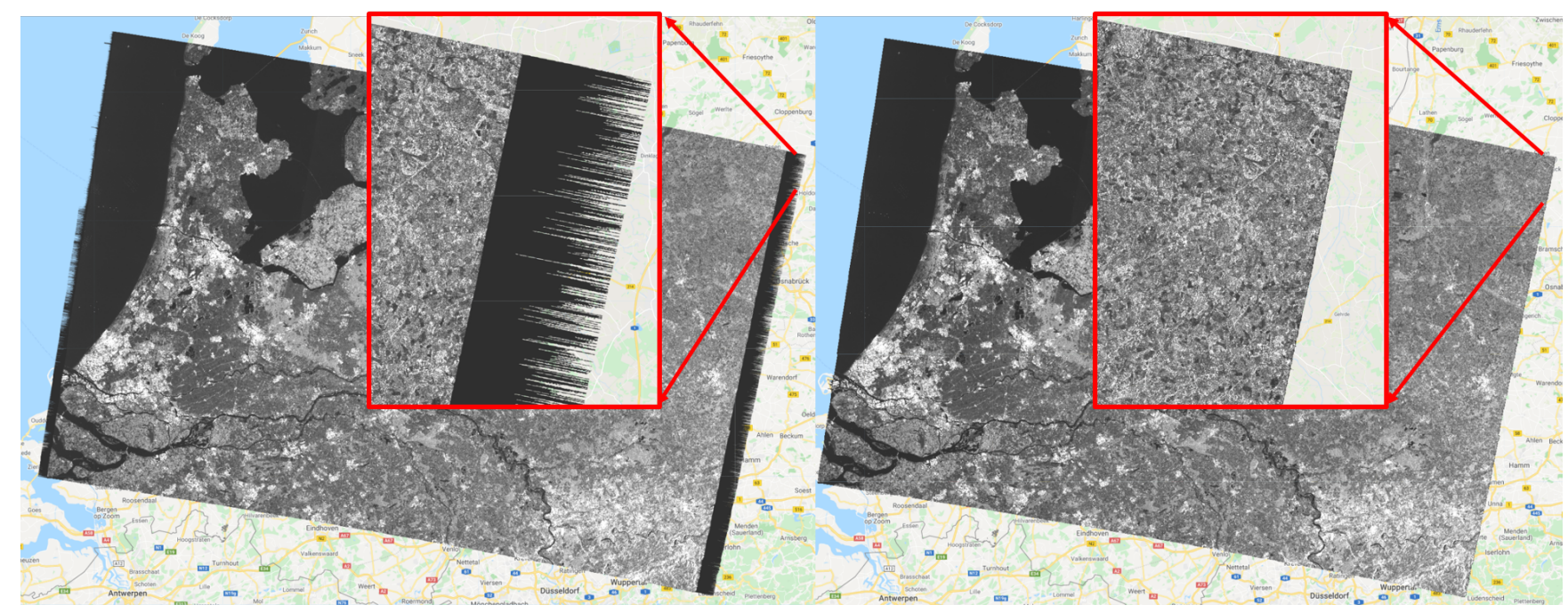

Figure 4. Border noise removal example for January 2018, orbit 37 from Sentinel 1A GRD (VH). The left image shows an image before border noise masking and the right image shows the image after masking. 

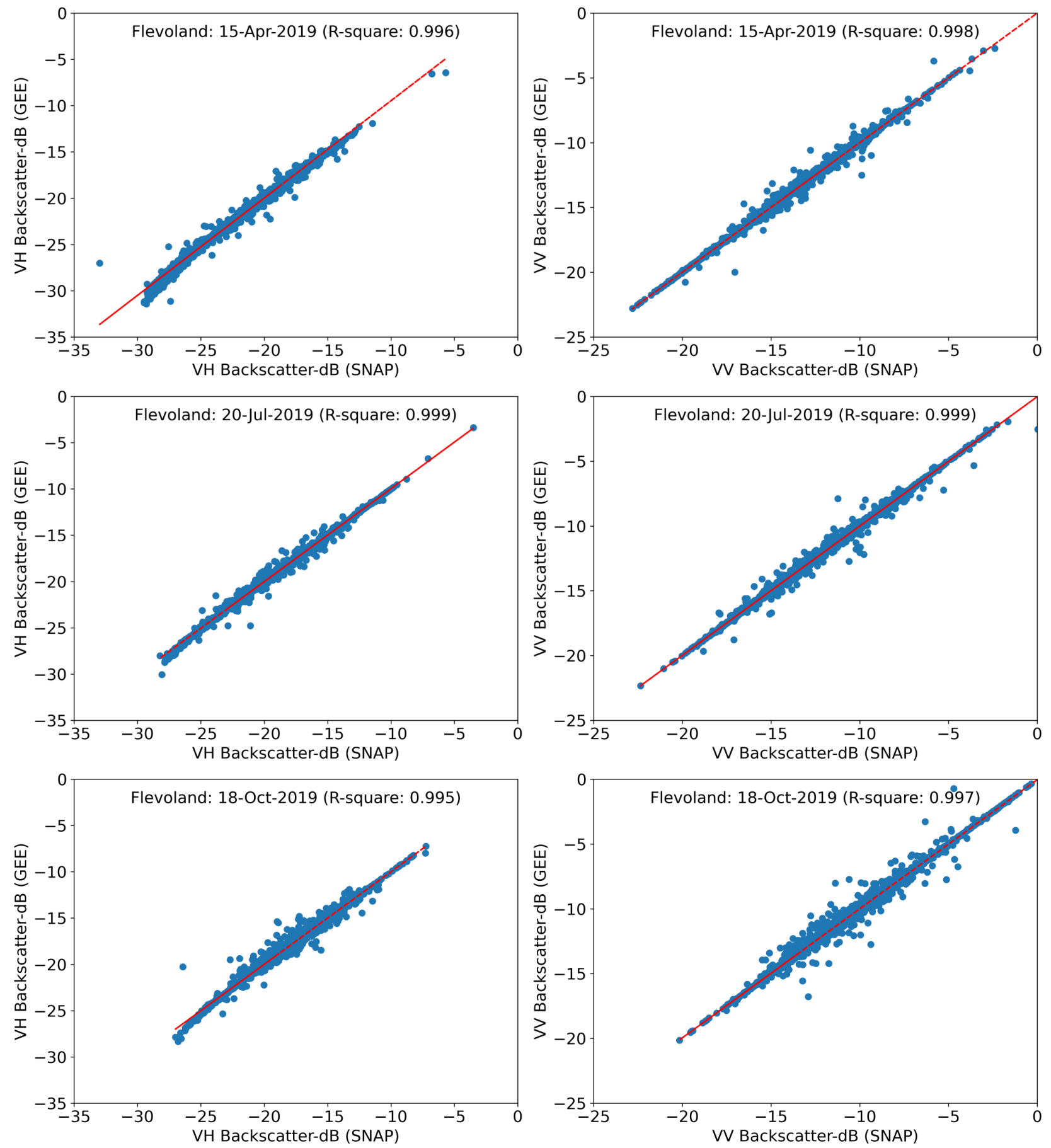

Figure 5. Comparison between GEE and SNAP extracted VH and VV backscatter values of Sentinel-1 GRD data acquired on 15 April 2019, 20 July 2019 and 18 October 2019 (RO-37) for BRP parcels in the Flevoland area. Every point in the scatter plots represent averaged value of one parcel. 


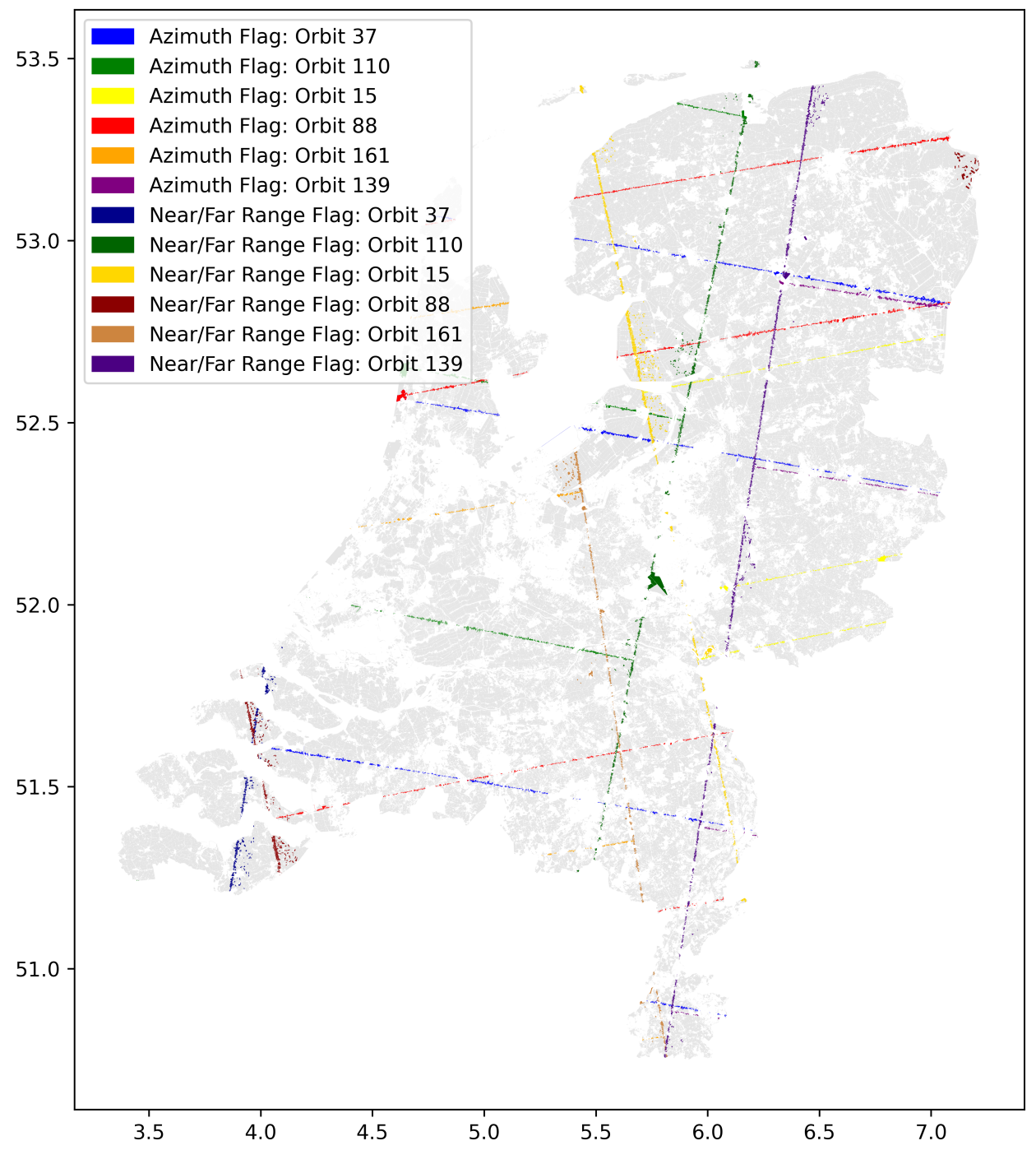

Figure 6. Flagged fields over the whole Netherlands for 6 RO covering the Netherlands $(15,37,88,110,139$ and 161) marking fields on the azimuth or near/far range image border. 

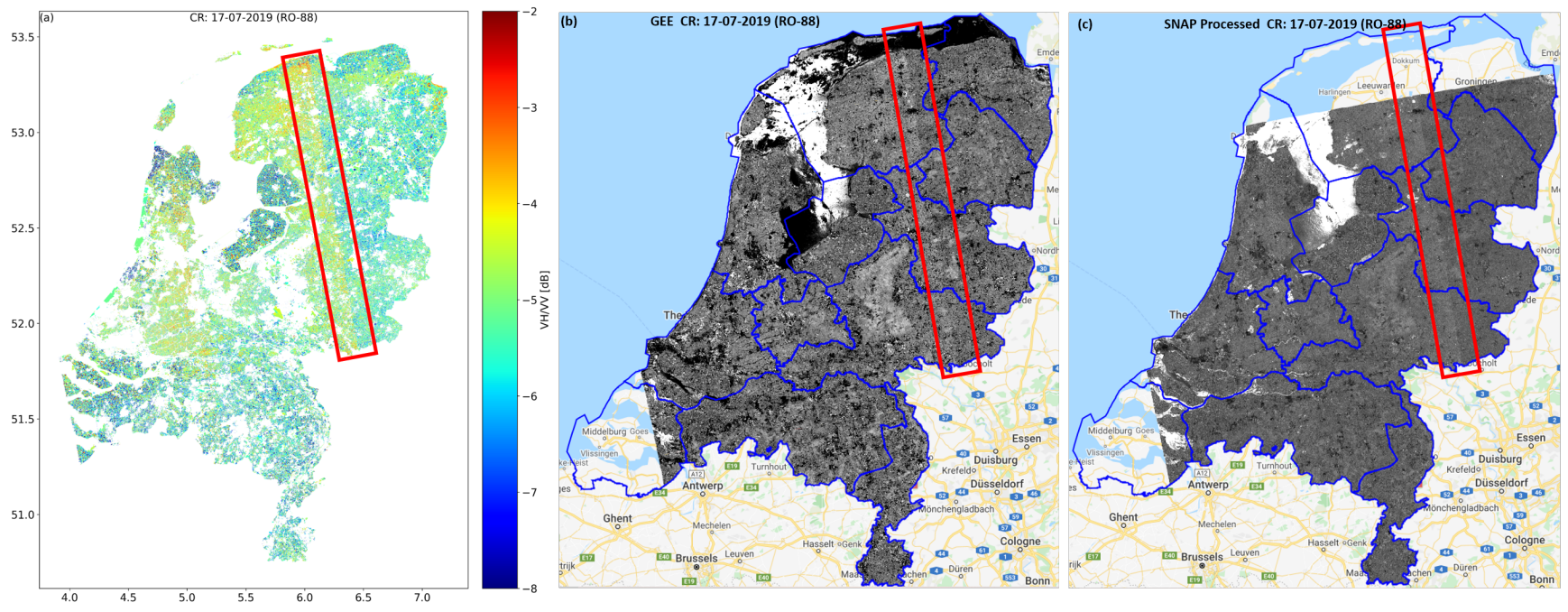

Figure 7. The Figures illustrate interswath granularity due to thermal/system noise in VH/VV (CR) ratio in (a) Output database (b) GEE Ingested Sentinel-1 GRD image processed for CR calculation (c) SNAP processed Sentinel-1 GRD image processed for CR calculation. All images show the CR on the 17-07-2019 for RO 88. The blue outlines in Figure (b), (c) show the boundaries of the dutch provinces. The red polygons highlight the jump in CR values observed for all cases.
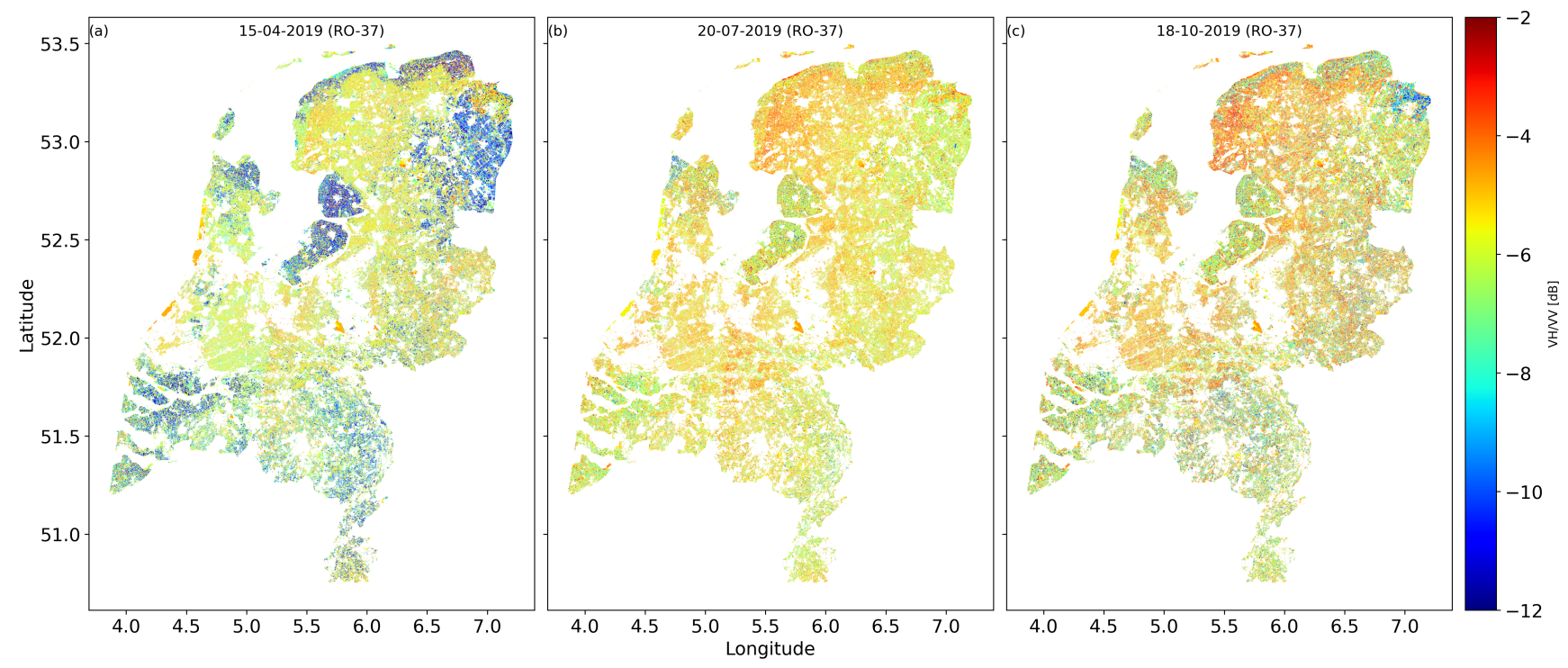

Figure 8. This Figure illustrate the spatial dynamics of VH/VV ratio for all the BRP parcels of the Netherlands for three dates (15-04-2019, 20-07-2019 and 18-10-2019) in RO 37. 

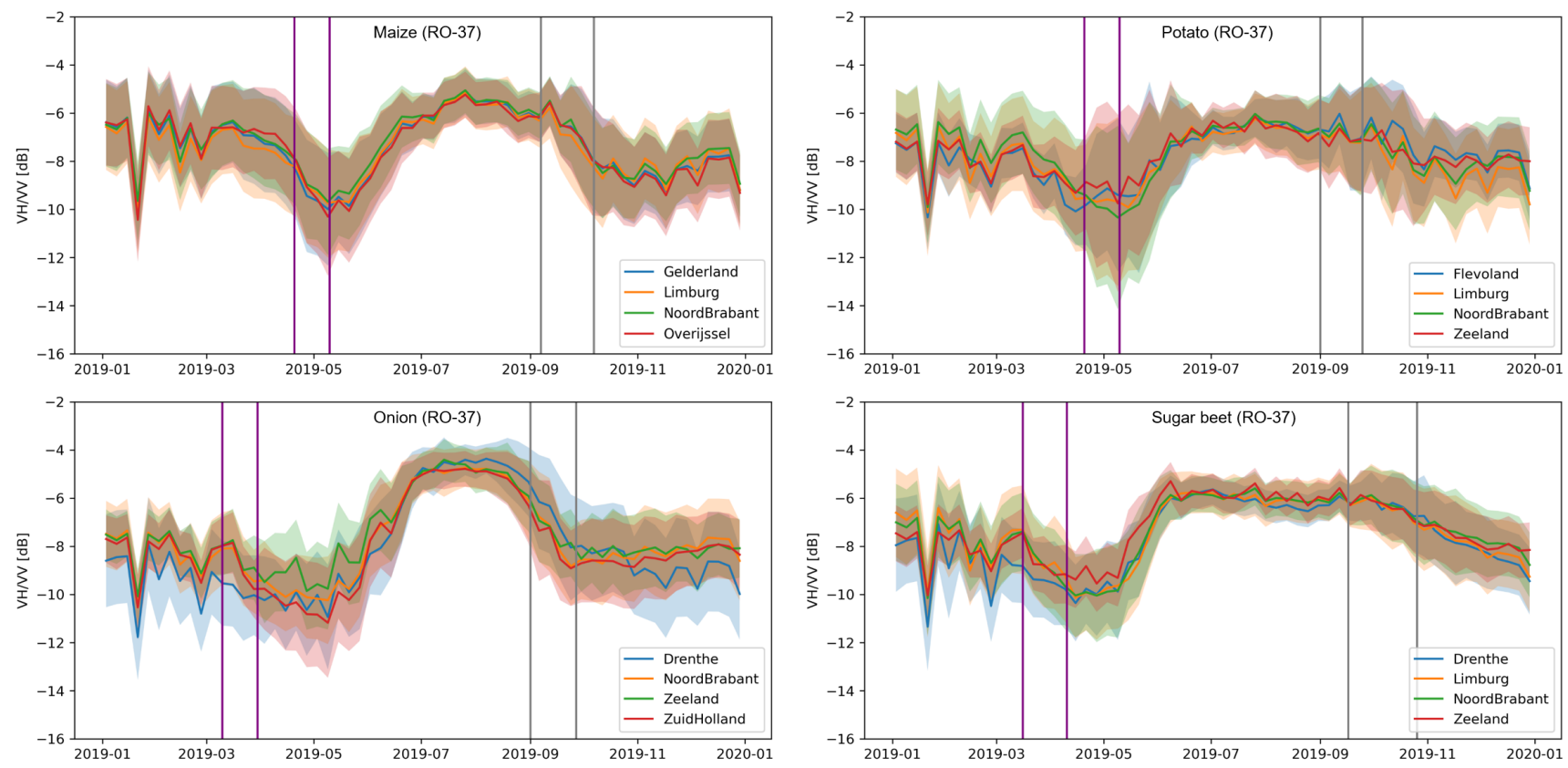

Figure 9. Time series of $\mathrm{VH} / \mathrm{VV}$ ratio for all the maize, onion, potato, and sugar beet parcels in four provinces. The solid line indicate mean and shaded areas indicate standard deviation values in four different provinces selected according to the maximum no. of crop parcels. Purple and gray vertical bars indicate transplanting and harvest period, respectively.
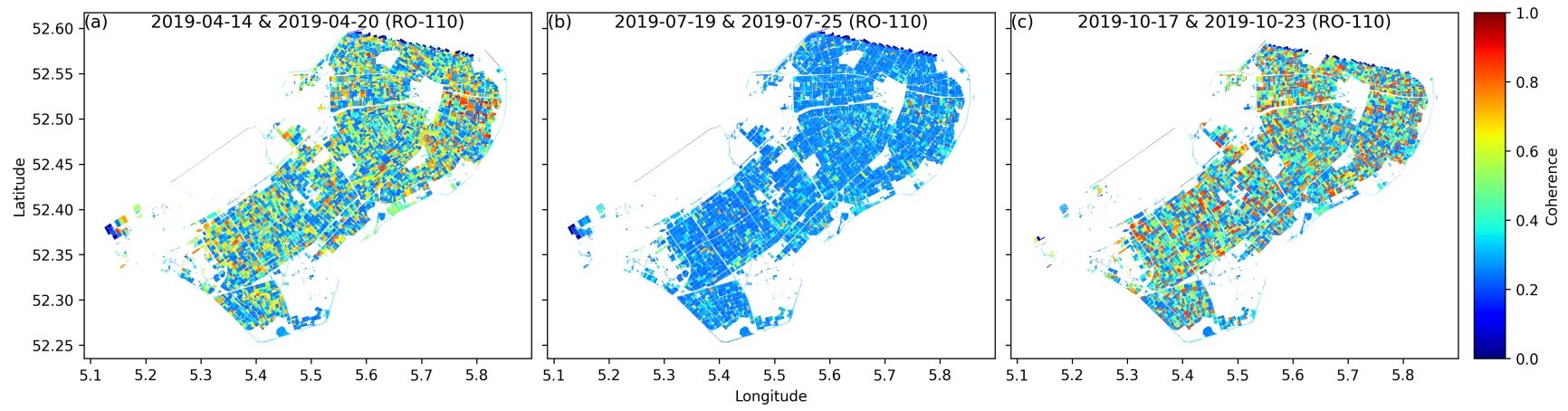

Figure 10. 6 day parcel-level interferometric coherence (VV channel) of Flevopolder region of the Netherlands for three time intervals: (a) 2019-04-14 \& 2019-04-20, (b) 2019-07-19 \& 2019-07-25, (c) 2019-10-17 \& 2019-10-23. Sentinel-1 SLC data in relative orbit 110 is used to generate coherence database. 


\begin{tabular}{|l|l|}
\hline Sentinel-1 & Data specifications in GEE \\
\hline Product type & Ground Range Detected (GRD) \\
\hline Data availability & Since April 2014 \\
\hline Acquisition mode & IW (Interferometric Wide), EW (Extra Wide) and SM (Strip Map) \\
\hline Polarizations & VV, HH, VV+VH and HH+HV \\
\hline Spatial resolution (posting) & 10,25 and 40 meter \\
\hline Thermal noise removal & After July 2015 acquisitions \\
\hline Border noise removal & After March 2018 acquisitions \\
\hline
\end{tabular}

Table 1. Specification of Sentinel-1 SAR data hosted at GEE platform.

\begin{tabular}{|l|l|l|}
\hline No. & Variable & Description \\
\hline 1 & Time & Acquisition time of the SAR image \\
\hline 2 & Longitude & Longitude of the parcel centroid \\
\hline 3 & Latitude & Latitude of the parcel centroid \\
\hline 4 & VV_mean & Average VV polarization backscatter intensity over parcel \\
\hline 5 & VV_std & Standard deviation VV polarization \\
\hline 6 & VH_mean & Average VH polarization backscatter intensity over parcel \\
\hline 7 & VH_std & Standard deviation VH polarization backscatter over parcel \\
\hline 8 & CR_mean & Average Cross-Pol-Ratio (VH/VV) backscatter intensity over parcel \\
\hline 9 & CR_std & Standard deviation Cross-Pol-Ratio $($ VH/VV) backscatter intensity over parcel \\
\hline 10 & LIA & Local Incidence Angle (adjusted for local topography) \\
\hline 11 & EA & Approximate viewing incidence angle in the GEE S1 GRD product \\
\hline 12 & AZA & Azimuth angle \\
\hline 13 & OID & Object ID (unique per parcel) \\
\hline 14 & MID & Sentinel-1 satellite mission ID $(0=$ S1A and 1 = S1B) \\
\hline 15 & RO & Relative orbit tracks of Sentinel-1 used in database \\
\hline 16 & Pix & Pixel count of the selected parcel \\
\hline 17 & Flag & Flags assigned to border parcels for each relative orbit \\
\hline
\end{tabular}

Table 2. List of variables and their description in the SandboxNL netCDF product. The backscatter intensities of VV, VH and $\mathrm{CR}$ are stored in linear scale

\begin{tabular}{|l|l|l|l|}
\hline Year & BRP parcel counts & Sentinel-1 images processed & Output database size ( GB) \\
\hline 2017 & 785710 & 1023 & 4.07 \\
\hline 2018 & 774822 & 1030 & 3.99 \\
\hline 2019 & 772565 & 1034 & 3.85 \\
\hline
\end{tabular}

Table 3. Details of Sentinel-1 images processed and output database size for a given year. 